\title{
OPTIMIZING THE STRUCTURE OF AMPHIPHILIC INVERTIBLE POLYMERS (AIPs) MADE OF PEGs AND FATTY COMPOUND SEGMENTS TO OBTAIN A SINGLE CRITICAL MICELLE CONCENTRATION
}

\author{
DANIELA ANDRADE-ACUÑA ${ }^{1,4}$, MOHAMED DAHROUCH ${ }^{1, *}$, SUSANA A. SANCHEZ ${ }^{2}$, ENZO DÍAZ ${ }^{1}$ \\ AND STEPHANE MAZIÈRES ${ }^{3}$
}

\author{
${ }^{I}$ Departamento de Química Orgánica, Facultad de Ciencias Químicas, Universidad de Concepción, 129 Edmundo Larenas, Concepción, Chile. \\ ${ }^{2}$ Departamento de Polímeros, Facultad de Ciencias Químicas, Universidad de Concepción, Chile. \\ ${ }^{3}$ Laboratoire des IMRCP, Université de Toulouse, CNRS UMR 5623, Université Paul Sabatier, 118 route de Narbonne, 31062 Toulouse Cedex 9, France. \\ ${ }^{4}$ Centro de Docencia Superior en Ciencias Básicas, Universidad Austral de Chile, Sede Puerto Montt, Los Pinos s/n. Balneario Pelluco. Puerto montt, Chile.
}

\begin{abstract}
Amphiphilic biopolyesters containing hydrophilic segments (PEGs) and hydrophobic blocks (silicon fatty from a castor oil derivative) showed the ability to selfassembly in inverted micelles. Due to their capability to form also direct micelles, these biopolyesters could be classified as amphiphilic invertible polymers AIPs. The micellar concentrations CMC and ICMC corresponding to the direct and inverted micelles respectively precisely correlated with PEG length. The methodology used in this investigation allowed to determine the PEG length needed to obtain the adequate structural biopolyester able to self-assembly in direct and inverted micelles from a unique concentration. Inverted micelle diameters, determined by DLS analysis, increased as the molecular polarity of the biopolyesters decreased. No individual inverted micellar were observed by TEM technique due to the concentration change during the sample preparation, however micellar macromolecular aggregations were revealed.
\end{abstract}

Keywords: Biopolyesters, Amphiphilicity, Fatty compound, PEGs, inverted micelles.

\section{INTRODUCTION}

Biomaterials have been widely studied in recent years, due to their role in various relevant areas, especially in agriculture and medicine. Most of the studies concern hydrogels and fibres, due to their potential application as fertilizers, carriers and tissue engineering [1-7]. Other promising biomaterials are micelles, for their biologic applications in encapsulating, carrying and releasing drugs [8-15].

In the last decades, the design and synthesis of polymers with amphiphilic properties have attracted vast scientific attention, because of their ability of selfassembling in aqueous medium forming different kinds of structures suitable for biologic applications[16-20]. In the process of micelle formation, the presence of polar and non-polar segments in the macromolecules creates a nucleus able to retain or encapsulate specific molecules for a later release. Molecular interactions between these polar and non-polar segments with the solvent explain micelle formation, which can theoretically take place in a polar or non-polar medium leading to direct or inverted micelles structures, respectively. In a polar and protic solvent like water, molecular interactions with the polar segments of the polymer usually favour the formation of direct micelles, where hydrogen bond is considered to be the main driving force to obtain such molecular buildings [2123]. On the other side, the formation of an inverted micelle obtained in an apolar media, may present some difficulties because of solvophobic interactions and a low dielectric constant the medium[24-26]. Amphiphilic polymers, known also as amphiphilic invertible polymers (AIPs), can self-assemble in either direct or inverted micelles, and they are very interesting materials for biologic applications such as drug delivery. Indeed, in a biologic organism, drugs having a certain efficiency potential, should be able to circulate in an aqueous medium and cross the lipid membranes (less polar environment) of the target cells. Therefore, polymers such as AIPs, capable of form direct and inverse micelles, should be considered as remarkable carriers able to both encapsulate and release host molecules: direct micelles would allow to encapsulate and carry the drugs and after contacting the lipid membranes, they will convert into inverted micelles by conformational inversion of polymer macromolecules, leading to the liberation of drugs inside of the cells [27-28]. The macromolecular design of AIPs for these bio-applications suggests that to obtain polar and non-polar segments in the same molecules, the use of biocompatible monomers is needed in the synthesis of the final macromolecule. PEGs, known for their non-toxicity and biocompatibility, are usually used for the polar block. For the non-polar block, the use of a fatty monomer seems to be the most suitable, aiming for the optimization of the molecular interactions with the lipid membrane [29]. Thinking in future biological applications of these AIPs, a relevant questioning attracts our attention, about how to make the AIPS concentration choice allowing to obtain both direct and inverted micelles if they present different micellar concentration CMC. Considering that the micelle formation usually starts above the critical micellar concentration (CMC), it would be possible to imagine using a concentration higher than the highest CMC, however, molecular aggregation could occur instead of micellar formation, in such concentration condition. Therefore, the strategy adopted to solve this challenge would orientate to find the adequate structural AIPS capable of self-assembly in direct and inverted micelles from a unique concentration. This paper describes a simple methodology, original as far we know, allowing determining such ideal molecular structure corresponding to AIPs formed of PEGs and fatty segments.

Recently, we reported the synthesis and the characterization of novel amphiphilic polyesters, composed of PEG and silicon fatty compound segments [30]. The fatty moiety was incorporated using a dimethyl ester monomer obtained by chemical modification (silylation) of the undecenoate methyl ester, considered to be an attractive derivative of castor oil for the preparation of biopolymers. Using polytransesterification reaction between PEGs of different molecular weight and the silicon fatty ester, biopolyesters presenting different grades of amphiphilicity were obtained, and they all showed the ability to selfassembly in direct micellar structures with an encapsulation potential. It is important to underline that, by increasing the length of the PEGs segments, the diameter of these biomaterials also increased, in a range of 70-190 nm, offering a potential way to control drug concentrations.

This article present two main purposes: One is to demonstrate that the biopolyesters described previously[30] are also able to self-assembly in inverted micelles if placed in an apolar environment, and thus, they could be classified as AIPs biomaterials. The second is orientated to describe a methodology to determine the right AIPS structure capable of self-assembly both direct and inverted micelle from a unique micellar concentration. The determination of inverted critical micellar concentration (ICMC) by fluorescence technique will be discussed compared with those reported for the direct micelle generated from the same biopolyesters. Additionally, analysis using Dynamic Light Scattering (DLS) and Transmission Electron Microscopy (TEM) will be used to confirm the micelles formation and estimate the diameters of these biomaterials.

\section{MATERIALS AND METHODS}

All the chemical reagents, including solvents, were purchased from SigmaAldrich and used without any additional purification. The biopolyesters (figure 1), used in this investigation are represented as PEG $_{\mathrm{XxX}}$-FASi (PEG for polyethylenglicol segment, $\mathrm{xxx}$ for the PEG molecular weight, FASi for the silicon fatty acid segment prepared from castor oil derivative). 
These biopolyesters were prepared following a reported procedure [30], applying a time polymerization of two hours.<smiles>[Y]CC(C)(C)OCCOC(=O)OCC[Si](C)(C)O[Si](C)(C)[GeH]C(C)=O</smiles>

Figure 1. structure of biopolyesters PEGxxx-FASi

The formation of an inverted micelle was performed employing a solvent mixture containing chloroform/ methanol in a volume ratio of 9:1 respectively. The inverted critical micellar concentration (ICMC) of each biopolyester was determined via steady-state fluorescence spectroscopy using pyranine (8Hydroxypyrene-1,3,6-trisulfonic acid trisodium salt) as a polar dye probe. Dissolutions of biopolyesters in a chloroform/ methanol mixture of pyranine were prepared in a concentration range of 0.005 to $0.9 \mathrm{mg} / \mathrm{mL}$. Fluorescence emission spectra were performed in a fluorescence spectrometer (Photon Technology International Inc. QuantaMaster, PAIS)) in the range 410-460 nm with excitation wavelength at $406 \mathrm{~nm}$. The procedure allowed to calculate the ICMC value of each biopolyester, from the emission spectra plotting the fluorescence intensity ratio $430 / 424$ [nm] versus the biopolyester concentration [30].

Transmission electron microscopy (TEM) was carried out using a JEOL 1200EXII instrument, with an activation voltage of $120 \mathrm{kV}$. The sample preparation consisted of a solution with a concentration similar to the ICMC. A drop of this solution was placed on a carbon-coated copper grid, followed by staining with $2 \%(\mathrm{w} / \mathrm{v})$ uranyl acetate solution and drying at room temperature. The hydrodynamic diameter of the micellar particles was obtained by dynamic light scattering (DLS) using a Brookhaven 90Plus instrument. A 35-mW laser was applied at $532 \mathrm{~nm}$, and the Stokes-Einstein equation was used to determine diffusion coefficients for each solution

\section{RESULTS AND DISCUSSION}

The process of micelle formation is usually studied using fluorescent probes. In the case of direct micelles, the different procedures described in the literature are very similar[31]-[33], since the solvent used is water. However, the methodology employed to generate inverted micelles may be very different since it depends on the critical step of choosing the correct solvent (or a mixture of solvents) to create the nonpolar environment for a specific molecule. Besides, the polar fluorescent probe should also be chosen carefully. The solvent mixture used to form inverted micelles should contain a large amount of non-polar solvent and a small amount of a polar solvent. The polar solvent is the driven force for the incorporation of the polar fluorescent probe in the polar core of the inverted micelle. In the case of the biopolyesters PEG-FASi, we founded that the right experimental conditions consisted in using the pyranine as the polar fluorescent probe and the non-polar environment were achieved by using a mixture of chloroform/methanol in a volume ratio of 9:1 respectively. Important to notice the use of chloroform as the non-polar component of the mixture, this aprotic solvent with a relatively low polarity allows the dissolution of small quantities of polar solvents such as methanol. Besides, this solvent mixture could be considered to be similar to the plasma membrane, which is amphipathic, where the polarity depends on the type of phospholipid present in the bilayer [3435].

Dissolutions of each biopolyester in such solvent mixture containing pyranine were examined by fluorescence spectroscopy. As an example, Figure 2a shows the emission spectra for the biopolyester $\mathrm{PEG}_{1000} \mathrm{FASi}$. A clear shift of the emission wavelengths from 430 to $424 \mathrm{~nm}$ could be appreciated since the biopolyester concentration increased. Figure $2 \mathrm{~b}$ shows the graphical determination of the critical micellar concentration for inverse micelles (ICMC), using the emission intensities at 430 and $424 \mathrm{~nm}$ obtained for the pyranine at an excitation wavelength of $406 \mathrm{~nm}$. The intensity ratio (I430/I424) is plotted versus the logarithm of the biopolymer concentration and the intersection of the two straight lines showing different slopes corresponds to the ICMC of a given biopolyester. This spectral trend is identical to those obtained from the biopolyesters $\mathrm{PEG}_{1500}-\mathrm{FASi}, \mathrm{PEG}_{2000}-\mathrm{FASi}$ and $\mathrm{PEG}_{3000}-\mathrm{FASi}$ and the determined ICMC values are within a range of 0.087 to $0.012 \mathrm{mg} / \mathrm{mL}$ (Table 1)
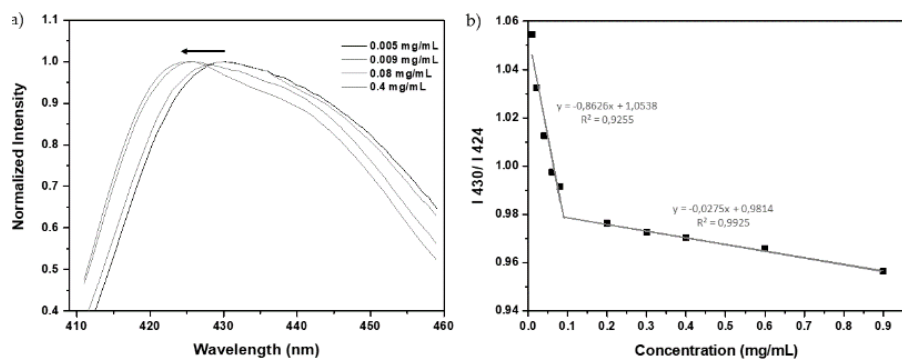

Figure 2. a) Emission spectra of an unsaturated solution of pyranine in chloroform/methanol in the presence of $\mathrm{PEG}_{1000}$-Fasi at different concentrations $(\mathrm{mg} / \mathrm{mL})$. b) Dependence of the intensity ratio (I430 / I424) of pyranine on the polymer concentration $(\mathrm{mg} / \mathrm{mL})$ for $\mathrm{PEG}_{1000}$-Fasi

Our results agree with the CMC values reported for PEG-FASi polymers and they are relatively low, meaning that a low concentration of biopolymers is required to create an inverted micelle structure. However, a clear tendency in the ICMC variation depending on the PEG length was observed. The ICMC values decreased when the PEG length increased (Table 1). This tendency could be explained considering the difference in polarity between the biopolyester and the environment. The small PEG segment in the biopolymer PEG $_{1000}-\mathrm{FASi}$, make this molecule more hydrophobic, favouring the good molecular affinity with the aprotic and nonpolar media composed of $90 \%$ chloroform. On the other extreme, $\mathrm{PEG}_{3000}-\mathrm{FASi}$, showing the highest polarity, does not exhibit such affinity with the solvent, causing the inverted micelle formation at lower ICMC. In the case of direct micelle formation, it is known that the CMC of the amphiphilic polymers decreases as the of hydrophobic segments increases [30], [33], [36] On the other hand, for inverted micelle formation, the increase of ICMC directly correlates with the growth of the hydrophobic segments.

Table 1. Hydrophobicity, weight average molecular weight $\mathrm{Mw}$, critical micellar concentration CMC, inverted critical micellar concentration ICMC and micelle size determined by DLS.

\begin{tabular}{|c|c|c|c|c|c|}
\hline polymers & $\begin{array}{c}\text { Hydrophobicity } \\
(\boldsymbol{\%})\end{array}$ & $\begin{array}{c}\text { Mw } \\
(\mathbf{g} / \mathbf{m o l}) \\
{[30]}\end{array}$ & $\begin{array}{c}\text { CMC } \\
(\mathbf{m g} / \mathbf{m L}) \\
{[30]}\end{array}$ & $\begin{array}{c}\text { ICMC } \\
(\mathbf{m g} / \mathbf{m L})\end{array}$ & $\begin{array}{c}\text { DLS } \\
(\mathbf{n m})\end{array}$ \\
\hline PEG $_{1000}$-Fasi & 33 & 31800 & 0.0062 & 0.087 & $116 \pm 5$ \\
\hline PEG $_{1500}$-Fasi & 25 & 28500 & 0.0064 & 0.057 & $85 \pm 3$ \\
\hline PEG $_{2000}$-Fasi & 20 & 31000 & 0.0070 & 0.033 & $61 \pm 2$ \\
\hline PEG $_{3000}$-Fasi & 14 & 31000 & 0.0094 & 0.012 & $52 \pm 5$ \\
\hline
\end{tabular}

a. hydrophobicity $\%$ containing in the monomeric unit -(PEG-FASi $)_{\mathrm{n}}{ }^{-}$.

Thinking on future biological applications of these new AIPs, a graphical comparison of critical micellar concentration for direct (CMC) and inverse micelles (ICMC) is shown in Figure 3. The graph shows that CMC and ICMC increased and decreased respectively as the PEG molecular lengths growth. In the case of the biopolyester showing a higher hydrophobicity, the difference between the $\mathrm{CMC}$ and the ICMC values was larger (Table.1). For example, the ICMC of the $\mathrm{PEG}_{1000}-\mathrm{FASi}$ was determined to be around $0.087 \mathrm{mg} / \mathrm{mL}$, whereas the CMC was around $0.0062 \mathrm{mg} / \mathrm{mL}$ [30]. Such a difference may represent a problem for biological applications, this fact could prevent the formation of one kind of micelle.

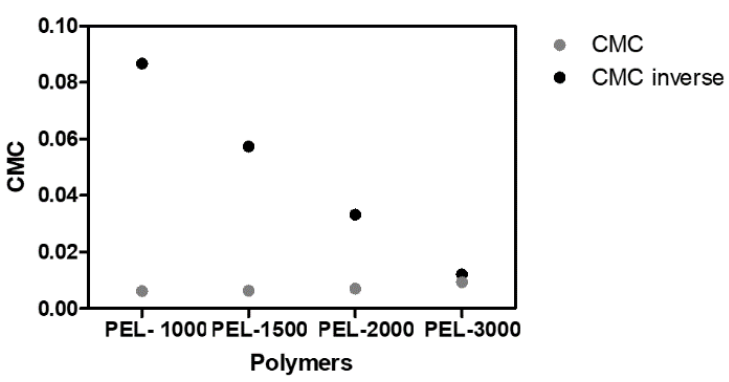

Figure 3. Comparison of CMC between direct and inverse micelles. 
For example, in the case of the biopolyester $\mathrm{PEG}_{1000}-\mathrm{FASi}$ (Figure 3) if the chosen concentration for a given micelle application is close to $0.0062 \mathrm{mg} / \mathrm{mL}$, the formation of direct micelles will occur whereas the formation of inverted micelle will be impossible. However, if the chosen concentration of the same biopolyester is around $0.087 \mathrm{mg} / \mathrm{mL}$, it will be possible to observe the formation of the inverted micelle, but the formation of direct micelle could be compromised by the genesis of molecular aggregation, due to the use of a concentration higher than the corresponding CMC. The key to avoiding changes in a micellar organization is to choose the adequate AIPs, the one having similar values of CMC and ICMC. As observed in figure 3, the difference between the $\mathrm{CMC}$ and ICMC decreases with the rise of the PEG molecular weight. Using a PEG with a molecular weight of $3000 \mathrm{~g} / \mathrm{mol}$, the biopolyester PEG $_{3000}-\mathrm{FASi}$ showed very close values of CMC and ICMC around 0.0094 and $0.012 \mathrm{mg} / \mathrm{ml}$ respectively. Thus, a graphical method to determine the molecular weight of a biopolyester that will easily change from direct to inverse micelles is to achieve the intersection of the two linear graphs in the type of plot shown in Figure 3. At this point, the molecular weight of the most suitable PEG could be estimated. In the case of the AIPS studied in this paper, the suitable molecular weight would correspond to a value of $3000 \mathrm{~g} / \mathrm{mol}$. Considering that the PEGs are chemical products available in numerous molecular weights, the strategy described in this paper to obtain biopolyesters able to form direct and inverted micelles from a unique concentration, seems to be very useful and straightforward for the study and applications of AIPs incorporating PEGs and fatty segments.

To complete the study of the inverted micelles, light dynamic scattering (DLS) and transmission electronic microscopy (TEM) were used to evaluate their dimensions. The sizes of the inverted micelles determined by DLS (Table 1) are comparable with those reported in the literature[30]. The micellar diameters, determined from dynamic light scattering analysis, decrease from 116 to $52 \mathrm{~nm}$, depending on the PEG molecular weight and consequently on the biopolyester polarity. It is well known that the size of a given macromolecular organization in a particular solution is related to the molecular interactions between the solvent and the polymer[37-38], therefore our results may be explained under this scenario. The larger size observed for the inverted micelles of biopolyester $\mathrm{PEG}_{1000}$-FASi could be explained by a higher intermolecular affinity between the fatty segments and the less polar environment. This interaction would be weak for the biopolyester $\mathrm{PEG}_{3000}-\mathrm{FASi}$, leading to the smaller inverted micelle obtained. It is also interesting to notice that the direct micelles generated in water present larger diameters as compared with the inverted micelle.

Transmission electron microscopy (TEM) was used to observe the inversed vesicles. However, for this technique, the sample needs to be warmed leading to partial evaporation of the solvent mixture (chloroform/methanol) and making the solution more concentrated. Therefore, it was not possible to realize analysis from a concentration close to the ICMC values. Unfortunately, no micelle structure could be observed at nanoscale. However, spherical shape structures can be observed at a micro-scale. TEM images showed possibly micellar aggregation due to a concentration higher than ICMC, preventing the observation of the molecular micelle organization. The aggregations showed a size close to $14 \mu \mathrm{m}$. The results of the TEM images of each biopolyester in an apolar environment were similar, and figure 4 shows the one corresponding to the biopolyester $\mathrm{PEG}_{2000}$-FASi.

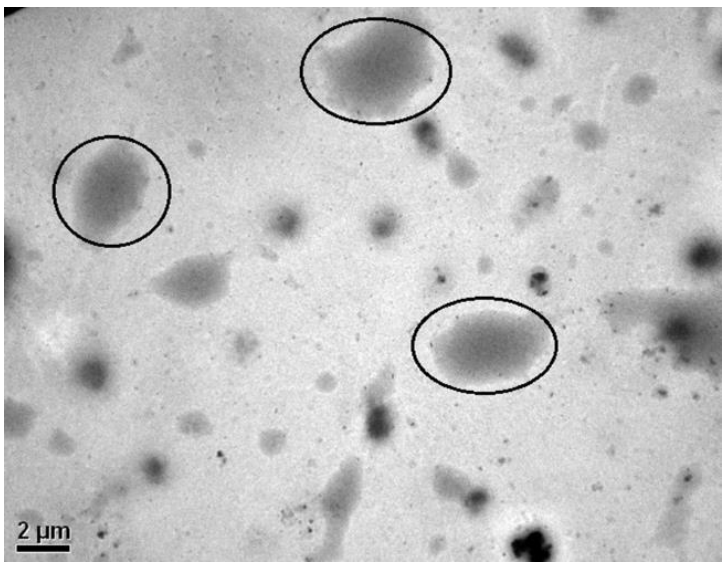

Figure 4. TEM image for $\mathrm{PEG}_{2000}$-Fasi.
Additionally, it is interesting to notice that our results can be explained in a model considering that direct micelles the hydrophilic segment (PEG) would strongly interact with water and favour the hydrogen bond formation. The macromolecule would have the freedom of movement and could expand easier in solution (Figure 5). Instead, the same biopolyester in an apolar medium, the PEG block would be located at the centre of the micelle without freedom of movement (probably forming a ball) leading to a micelle of smaller size. This model could also explain how the same macromolecule may interconvert from direct to reverse micelles with the change of medium polarity and be a good candidate for drug carrier and delivery.
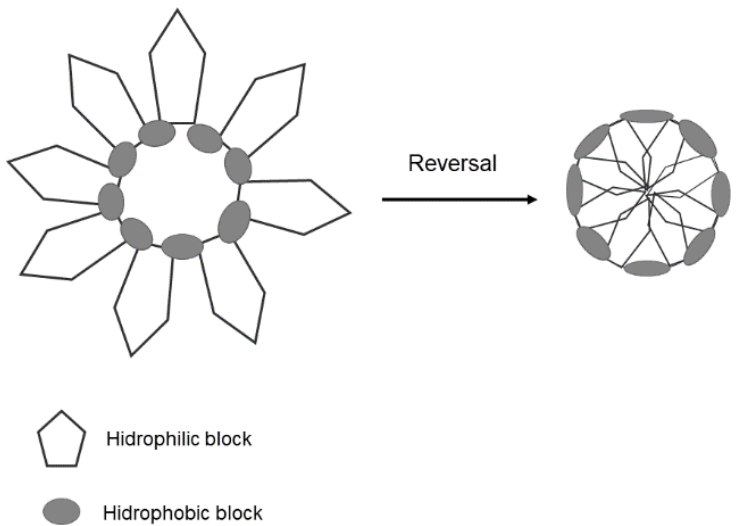

Figure 5. Diagram for the proposed model explaining the inversion of micelle from a polar medium to a non-polar medium.

\section{CONCLUSIONS}

An attractive macromolecular class of AIPs was found through the chemical study of amphiphilic biopolyesters containing PEGs and a silicon fatty compound obtained from a castor oil derivative. The inverted micelle formation was studied by fluorescence techniques, after setting up successfully the adequate experimental conditions. Considering that, for future applications in drug delivery, the direct and inverted micelle formation should take place using the same micellar concentration of these biopolyesters PEG-FASi, it was very relevant to determine the most suitable biopolymer structure, displaying same values of CMC and ICMC. As the FASi hydrophobic segment did not change, it was interesting to find a linear correlation between CMC or ICMC versus the PEG hydrophilic segment length. Consequently, by analyzing this linear correlation, it was possible to determine the right PEG length which should be used in the synthesis, to achieve appropriate biopolymer structure able to form direct and inverted micelles using the same initial concentration. This simple methodology described in this paper, original as far as we know, could be applied to other linear AIPS formed from the hydrophilic PEGs segment, which maintain in the structure the same hydrophobic segment. Concerning the inverted micelle size using DLS analysis, the study confirms that the intermolecular interaction between the amphiphilic polymers and the solvent could be considered as a key effect to understand the important diameter differences if compared to those obtained from direct micelles. Although the TEM could not show individual micelle formation due to experimental conditions, the results exhibited by this technique indicate possible spherical micellar aggregation and encourage us to investigate such macromolecular formation. Finally, based on these results described in this paper, the biopolyesters PEG-FASi represent an attractive class of AIPs to be studied for future applications orientated to demonstrate their drug delivery properties.

\section{FUNDING}

This study was supported by the project VRID 216.023.052-1.0 and the "Dirección de Investigación e Innovación de la Facultad de Ciencias Químicas".

\section{ACKNOWLEDGEMENTS}

The authors thank "Universidad de Concepción" for their academic support.

\section{CONFLICTS OF INTEREST}

The authors declare no conflict of interest. 


\section{REFERENCES}

1. S. Van Vlierberghe, P. Dubruel, E. Schacht, Biomacromolecules 12, 1387, (2011).

2. R. Zarzycki, Z. Modrzejewska, K. Nawrotek, U. Lek, Ecol. Chem. Eng. S. 17, 117, (2010).

3. T. Zheng, Y. Liang, S. Ye, Z. He, Biosyst. Eng. 102, 44, (2009).

4. F. Brandl, F. Kastner, R. M. Gschwind, T. Blunk, J. Teßmar, A. Göpferich, J. Control. Release 142, 221, (2010).

5. N. S. Malik, M. Ahmad, M. U. Minhas, PLoS One 12, 1, (2017).

6. N Katir, D. Andrade,M. Dahrouch, E. Diaz, N. Gatica, D. Hourlier, N. Reyes, M. Zarraga, J. Chil. Chem. Soc. 1, 2784, (2016).

7. M. R. Shahid Bashir, Y. Y. Teo, S. Ramesh, K. Ramesh, A. A. M. Rizwan, J. Chil. Chem. Soc. 64, 2, (2019).

8. B. A. Laurent, S. M. Grayson, Polym. Chem. 3, 1846, (2012).

9. L. Yin, Y. Chen, Z. Zhang, Q. Yin, N. Zheng, J. Cheng, Macromol. Rapid Commun. 36, 483, (2015).

10. N. M. Correa, J. J. Silber, R. E. Riter, N. E. Levinger, Chem. Rev. 112, 4569, (2012).

11. G. N. Smith, P. Brown, S. E. Rogers, J. Eastoe,Langmuir 29, 3252, (2013).

12. S. Taokaew, M. Ofuchi, T. Kobayashi, Materials (Basel) 12, 1160, (2019).

13. L. P. Sze, H. YinLi, K. L. A. Lai, S. F. Chow, Q. Li, K. W. KennethTo, T. N. T. Lam, W. Y. T. Lee, Colloids Surfaces B Biointerfaces 184, 110554, (2019).

14. X. Zhang, N. Liang, X. Gong, Y. Kawashima, F. Cui, S. Sun, Colloids Surfaces B Biointerfaces 177, 11, (2019).

15. R. G. Thomas, M. J. Moon, J. H. Kim, J. H. Lee, Y. Y. Jeong, PLoS One 10, $1,(2015)$

16. B. Zhang, H. Zhang, Y. Li, J. N. Hoskins, S. M. Grayson, ACS Macro Lett. 2, 845, (2013).

17. D. Abdelhamid, H. Arslan, Y. Zhang, K. Uhrich, Polym. Chem. 5,1457, (2014).
18. G. B. Behera, B. K. Mishra, P. K. Behera, M. Panda, Adv. Colloid Interface Sci. 82, 1, (1999).

19. T. Yang, W. Li, X. Duan, L. Zhu, L. Fan, Y. Qiao, H. Wu, PLoS One 11, 1, (2016).

20. H. C. Andrés, F. Olea, J. Villena, A. Moller, R. Martínez, J. Chil. Chem. Soc. 64, 4437, (2019).

21. J. J. Silber, R. D. Falcone, N. M. Correa, M. A. Biasutti, E. Abuin, E. Lissi, P. Campodonico, Langmuir 19, 2067, (2003).

22. U. Anand, C. Jash, S. Mukherjee, J. Colloid Interface Sci. 364,. 400, (2011),

23. A. Harada, K. Kataoka, Polym J 50, 1, (2017).

24. S. Yi, F. Dai, C. Zhao, Y. Si, Sci. Rep 7, 1, (2017).

25. M. S. Akhter, Colloids Surfaces A Physicochem. Eng. Asp. 150, 25, (1999).

26. C. Reichardt and T. Welton, Solvents and Solvents Effects in Organic Chemistry, Fourth edi. Germany: Wiley-VCH, 2011.

27. A. Voronov, Biomacromolecules 13, 2537, (2012).

28. A. Maran, M. J. Yaszemski, A. Kohut, A. Voronov, materials 9, 10, (2016).

29. S. Ramadurai, A. Kohut, N. Kumar, O. Zholobko, J. Colloid Interface Sci. 542, 483, (2019).

30. D. andrade, C. Moya, F. Olate, N. Gatica, S. Sánchez, E. Díaz, E. Elgueta, M. Parra, M. Dahrouch, RSC Adv., 6, 38505 (2016).

31. J. W. Bae, E. Lee, K. M. Park, K. D. Park Macromolecules, 42, 3437, (2009).

32. F. Bougard, C. Giacomelli, L. Mespouille, R. Borsali, P. Dubois, R. Lazzaroni, Langmuir 24,8272, (2008).

33. Q. Cui, F. Wu, E. Wang, J. Phys. Chem. B 115, 5913, (2011).

34. C. L. Salcedo, A. M. Bouchet, M. A. Nazareno, E. A. Disalvo, M. A. Frias, Colloids Surfaces B Biointerfaces, 113, 243, (2014).

35. A. M. Bouchet, M. A. Frías, F. Lairion, F. Martini, H. Almaleck, G. Gordillo, E. A. Disalvo, Biochim. Biophys. Acta - Biomembr. 1788, 918, (2009).

36. L. Chen, T. Ci, L. Yu, J. Ding, Macromolecules 48, 3662, (2015).

37. B. Obermeier, H. Frey, Bioconjug. Chem. 22, 436, (2011).

38. N. Katir, A. El Kadib, M. Dahrouch, A. Castel, N. Gatica, Z. Benmaarouf, P. Riviere, Biomacromolecules 10, 850, (2009). 